\title{
Importance of Allergic Hypersensitivity to Medications
}

\section{Angel San Miguel-Rodríguez ${ }^{1 *}$, Alicia Armentia ${ }^{2}$, Sara Martín-Armentia ${ }^{3}$, Blanca Martín-Armentia ${ }^{1}$, $M^{a}$ Carmen Lozano Estevan ${ }^{4}$ and Irene Iglesias Peinado ${ }^{5}$}

\author{
${ }^{1}$ Research Support Unit, Hospital Universitario Río Hortega, Valladolid University, Spain \\ ${ }^{2}$ Allergy Department, Hospital Universitario Río Hortega, Valladolid University, Spain \\ ${ }^{3}$ Paediatric Department, Hospital Rio Carrión, Spain \\ ${ }^{4}$ Pharmacy Faculty, Universidad Alfonso X el Sabio, Spain \\ ${ }^{5}$ Pharmacy Faculty, Universidad Complutense, Spain
}

*Corresponding author: Angel San Miguel-Rodríguez, Research Support Unit, Hospital Universitario Río Hortega, Valladolid University, C/San Antonio de Padua 1, 1Dcha, 47003 Valladolid, Spain, E-mail: asanmi@saludcastillayleon.es

\begin{abstract}
Allergic drug reactions (ADR) are the third most common reason for consultation in allergy services. Epidemiological data are imprecise. Globally, ADR affect $10-20 \%$ of hospitalized patients and up to $7 \%$ of outpatients. However, this might be an underestimate due to underreporting, or an overestimate due to unexplained reactions often being classified as "allergic".

Being labeled as "allergic" carries risks for the patient and concerns for the physician when it comes to drug therapies. On the one hand, the possibility of anaphylactic shock, but on the other hand, the use of therapies that are not first choice treatments and are usually more expensive, have more side effects and an increased risk of treatment failure. ADR also increase the costs of medical care due to increased use of emergency services, hospitalizations, suspension of tests and extended hospital stays. Quantification of the risk of an allergic drug reaction through the medical history is essential in clinical decision making. However, in normal clinical practice, this evaluation is generally entirely subjective. The present revision makes a summary that the symptoms, diagnosis, treatment and management of drug allergy and summarize the experience of our group.
\end{abstract}

\section{Keywords}

Drug allergy, Drug reactions, Illicit drug allergy, Allergic drug reactions

\section{Introduction}

A drug allergy, or an allergic drug reaction (ADR), is an adverse drug reaction that results from a specif- ic immunologic response to a medication. ADR are the third most common reason for consultation in allergy services [1].

Epidemiological data are imprecise. ADR affect 10$20 \%$ of hospitalized patients and up to $7 \%$ of outpatients [2]. However, this might be an underestimate due to underreporting, or an overestimate due to unexplained reactions often being classified as "allergic" [3]. In the Spanish Alergológica 2015 epidemiological study of ADR the diagnosis remained as suspected in $39.2 \%$ of active principles [1].

This carries risks for the patient. On the one hand, the possibility of anaphylactic shock: $13 \%$ of cases are due to drug allergies [4]. On the other hand, the use of therapies that are not first choice treatments and are usually more expensive [5], have more side effects and an increased risk of treatment failure [6-10]. ADR also increase the costs of medical care due to increased use of emergency services, hospitalizations suspension of tests and extended hospital stays [6-10].

Assessment by an allergy specialist is crucial since it allows a diagnosis to be reached in some cases and allergies to be ruled out in most patients. Complementary tests include skin tests and laboratory analysis. However, the sensitivity of these diagnostic methods is not optimal and, in any case, the definitive test to establish or exclude the diagnosis is a controlled exposure test [11].

Citation: Miguel-Rodríguez AS, Armentia A, Martín-Armentia S, Martín-Armentia B, Estevan MCL, et al. (2018) Importance of Allergic Hypersensitivity to Medications. Int J Aller Medications 4:032. doi. org/10.23937/2572-3308.1510032

Accepted: August 06, 2018: Published: August 08, 2018

Copyright: (C) 2018 Miguel-Rodríguez AS, et al. This is an open-access article distributed under the terms of the Creative Commons Attribution License, which permits unrestricted use, distribution, and reproduction in any medium, provided the original author and source are credited. 
This may be expensive, time consuming and potentially dangerous, but is usually necessary, as no sensitization to the drug involved is found in two-thirds of patients with suspected ADR [11].

The clinical history is essential when deciding whether to submit patients to controlled drug exposures. Such testing is necessary to rule out sensitization but can be costly and has some risks $[12,13]$.

Quantification of the risk of ADR by the medical history is vital to decision making by the allergy consultant, and to provide criteria for other physicians to refer the case to a specialist. It may also allow an estimate of the accuracy of suspected diagnoses. The present revision makes a summary that the symptoms, diagnosis, treatment and management of drug allergy and summarize the experience of our group [14,15].

\section{Management of Drug Allergy}

An allergic reaction can occur when the immune system overreacts to a harmless substance, in this case a drug, which triggers the allergic reaction. Sensitivities to drugs may produce similar symptoms, but do not involve the immune system. Adverse reactions to drugs are common, but everyone responds differently. One patient may develop hives when taking a certain medication, while another person on the same medication may have no adverse reaction. Only $5-10 \%$ of these reactions are due to an allergy response. The chances of developing an allergy are higher when the medication is taken frequently or when is rubbed on the skin or given injection, rather than taken by mouth [16].

It has been postulated that, unlike other allergic diseases, genetic influences are not so important in the case of ADR and that sensitization is principally due to exposure [17].

Certain medications are more likely to produce allergic reactions than others. The most common are: antibiotics, the most prevalent penicillin and into these group the amoxicillin. The second group is aspirin and non-steroidal anti-inflammatory drugs as Ibuprofen and metamizole. The third group includes chemotherapy and monoclonal antibody therapy $[13,14,18,19]$.

NSAIDs are the drugs most commonly implicated in studies on hypersensitivity reactions, followed by betalactamics antibiotics [20]. However, after adjustment for the variable "pharmacological class" Betalactamics were the major cause of allergies. The estimated proportion of true allergic reactions was 32\% for Betalactamics and less than $17.4 \%$ for NSAIDs [21].

In our series there was a majority of females. However, the difference between sexes with respect to confirmed diagnoses was not significant after adjusting in the multivariate analysis. This suggests there may be a considerable number of undiagnosed ADR in the general population, principally in males who have not sought consultation.
The latency period can be divided into three categories, "immediate" (occurred in the first hour), "late" (occurred later), and "unknown" (the patient did not remember the time elapsed).

Quantification of the risk of ADR by the medical history is vital to decision making by the allergy consultant, and to provide criteria for other physicians to refer the case to a specialist. It may also allow an estimate of the accuracy of suspected diagnoses.

\section{Symptoms of Drug Allergy}

Adverse reactions to drugs range from stomach ache with aspirin or diarrhea and hair loss with cancer chemotherapy or to upset stomach or diarrhea from antibiotics [3]. For example, ACE (angiotensin converting enzyme) inhibitors for high blood pressure, can produce cough or facial and tongue swelling. In many cases, it can be difficult to determine if the reaction is due to the medication or something else. This is because your symptoms may be similar to other conditions. It is frequently blamed the symptoms to medication that to foods allergens consumed by the patient in the same day. In our Hospital, the diagnostic panel we used in testing drug allergy include also drug excipients (soy, lactose, cornstarch..) and foods. It is very frequent than Ibuprofen acts as cofactor in Anisakis simplex allergy, for example.

The most frequent types of allergic symptoms to medication are: Swelling, such as in the face, skin rashes, hives, itching and respiratory problems.

Anaphylaxis is a resinous allergic response that involves swelling, hives, lowered blood pressure, and in severe cases, shock. If anaphylactic shock is not treated immediately, it can be fatal. A major difference between anaphylaxis and other allergic reactions is that anaphylaxis typically involves more than one system of the body.

Anaphylaxis requires immediate medical attention because the result can be fatal. The election treatment is epinephrine. Patients with anaphylaxis should be trained in auto-injectable epinephrine.

\section{Diagnosis of Drug Allergy}

Suspected diagnoses were more common than confirmed diagnoses [3]. This, together with the supposed prevalence of ADR, suggests that over-diagnosis is a public health problem. This is probably due to classification bias among professional observers, i.e., clinical variability due to differences in the medical history, the observation of patients and their diagnostic and/or therapeutic classification.

There are principal things to know: A description of the symptoms, when these began, how long the symptoms lasted and any other medications (and food) taken during this time, including over-the-counter drug and 
illicit drugs. A history of reactions to different medication, or a serious reaction to a drug ought to be studied by an allergist, that has specialized training and experience to diagnosis the problem and help the patients to develop a plan to protect him in the future.

We comment the anamnesis and diagnosis difficulties later.

\section{Treatment of Drug Allergy}

The first treatment after suspect that a drug allergy has occurred is to call the physician. If the symptoms are severe, seek medical help immediately [1-3].

An anaphylactic reaction requires immediate medical attention because the result can be fatal. In most cases of adverse reactions, the physician can prescribe and alternative medication. The drugs more often used to treat drug allergy are antihistamines, corticoids and epinephrine. Standardized allergy testing is available for all the drugs and may be followed by an oral challenge in the clinic. Such testing provided a high degree of reassurance that penicillin and like medicines can be tolerated in the future.

When no alternative is available and the medication is essential, a desensitization procedure to the medication may be recommended. This involves gradually in- troducing the medication in small doses until the therapeutic dose is achieved.

It is very important that the physician, dentist and pharmacy are kept current regarding possible drug allergies. This will help determine which medications should be avoided.

\section{Our Experience in Diagnosis of Allergic Drug Reactions}

We performed an observational study in which 696 active principles, corresponding to 466 patients aged > 14 years attending the Allergy Service of our University Hospital during a year, were included. Simple binary logistic regression was used to determine associations between variables from the medical history and the final diagnosis confirmed/ruled out [2].

The variables studied were sex, age at the time of reaction, relatives with allergic diseases, personal history of allergic disease, personal history of drug allergy, chronic disease, chronic treatment, skin tests to aeroallergens (pollen, mites, dander, mold), skin test to food allergens (flour, egg, milk, fish, fruit, nuts, legumes), IgE total value higher or lower than the IgE median of 60 found in our sample (IgE60), number of drugs assessed, time to assessment, referring service, emergency room

\section{6 active principles evaluated}

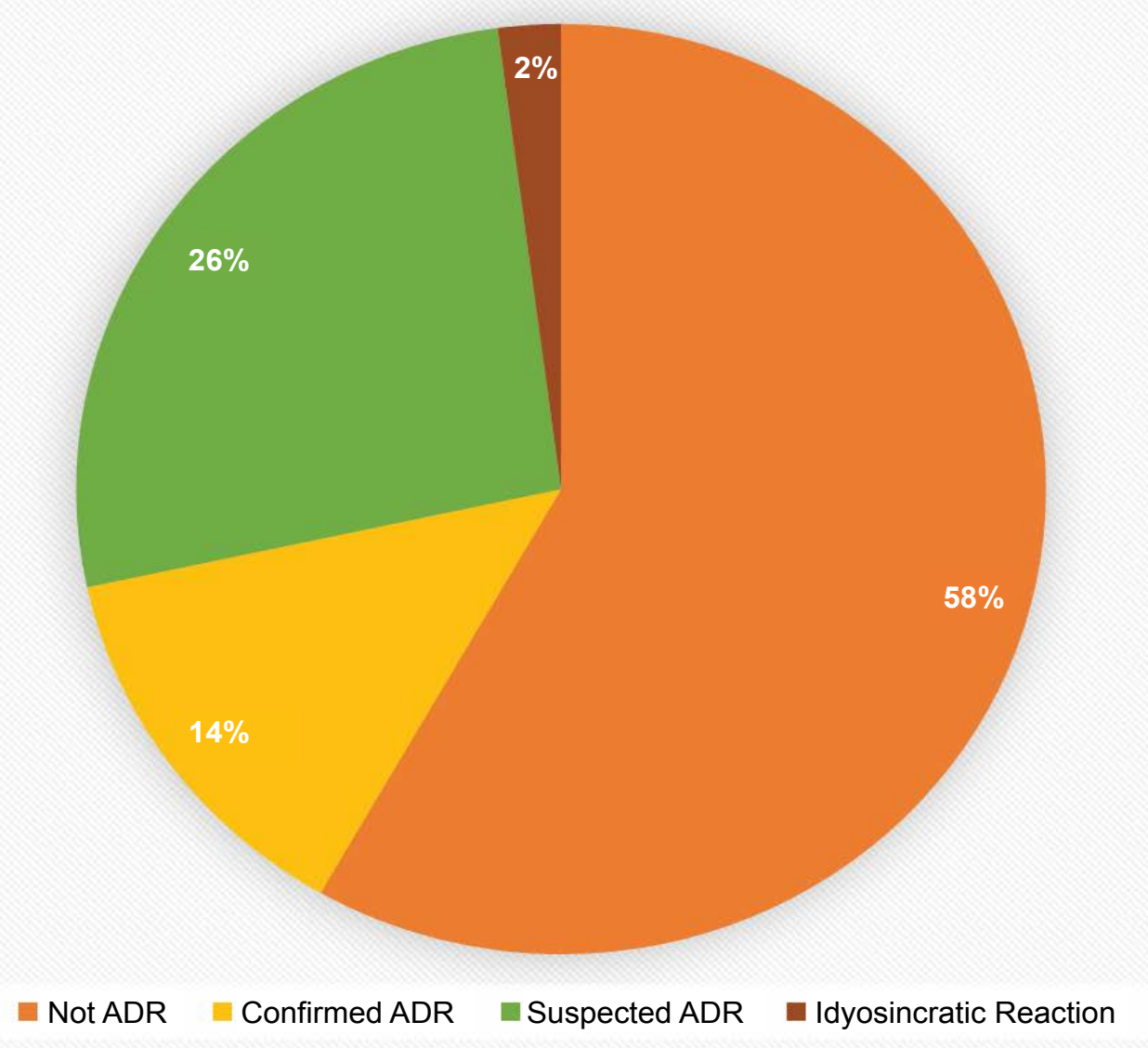

Figure 1: Of the 626 active principles finally evaluated, ADR was ruled out in $58.1 \%$ and confirmed in $13.4 \%$, while $26.4 \%$ remained as suspected in the remaining $2.1 \%$ of cases, a diagnosis of idiosyncratic reactions to NSAIDs was made. 
visits, hospitalization, specific drug to be assessed, route of administration, number of doses, clinical manifestations, duration of episode, latency period between exposure and reaction, number of episodes, and previous tolerance.

Various categories may be established. Confirmed diagnosis: when skin tests, specific IgE test or controlled exposure to the drug are positive. Diagnosis ruled out: When the controlled exposure test is negative. Suspected diagnosis: Suspected according to the medical history but no controlled exposure test is performed. The drug is withdrawn and an alternative drug is usually tested. Non-steroidal anti-inflammatory drugs (NSAIDs) often result in idiosyncratic reactions that are clinically very similar to allergic reactions. Therefore, two further categories can be established. Confirmed diagnosis of idiosyncratic reaction: when there is a positive oral controlled exposure test to aspirin, and Suspected diagnosis of idiosyncratic reaction: suspected according to the medical history, but no controlled challenge test is performed.

Variables useful in predicting a final diagnosis of allergic drug reaction were: age, sex, drug class, number of active principles, time to the reaction, number of doses, clinical presentation suggestive of allergic disease, and time to medical consultation. More than one suspected active principle was studied in $57.7 \%$ of patients. Of the 626 active principles finally evaluated, ADR was ruled out in $58.1 \%$ and confirmed in $13.4 \%$, while $26.4 \%$ remained as suspected (Figure 1). In the remaining $2.1 \%$ of cases, a diagnosis of idiosyncratic reactions to NSAIDs was made and confirmed by a controlled exposure test. With respect to the type of drug, 244 (39\%) were NSAIDs, 172 (27.5\%), penicillin antibiotics, and 210 $(33.5 \%)$ other drug classes (other antibiotics $11.4 \%$, anesthetics $3.5 \%$, corticosteroids $2.9 \%$, opiates $2 \%$, proton pump inhibitors $1.6 \%$, radio contrast agents $0.4 \%$, other drug classes $11.8 \%$ (Figure 2 ).

A case with a high probability of being confirmed as an ADR in the consultation would be an elderly man (e.g. 80 years) who reports a reaction involving only one active ingredient and which occurred within the first hour after exposure, with the consultation being made within the first year after the reaction, and with the initial dose of treatment being clearly suggestive of ADR, and the active principle being a betalactamics. True adverse drug reactions were estimated to occur in $20 \%$ of active principles.

Anaphylaxis was confirmed in only 1 active principle of all sample, by skin test, and had a high pretest score. However, non-allergic reactions could only be ruled out in $52.2 \%$ and $40 \%$ of active principles had a low probability pretest, $10 \%$ or less.

In summary, the use of mathematical models could greatly improve the discriminatory capacity of the medical history. The suspected diagnosis made by an aller-

\section{Type of drug involved in ADR}

300

250

200

150

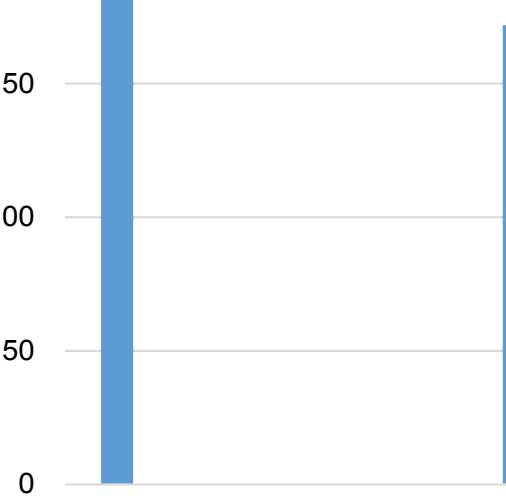

NSAIDs

Other Atibiotics

- Proton pump inhibitors

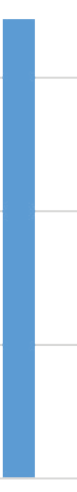

Penicilin

- Anesthesics

- Radio Contrast agents

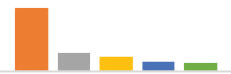

Other drugs

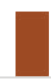

Corticosteroid $\quad$ Opiates

- Other

Figure 2: With respect to the type of drug, 244 (39\%) were NSAIDs, $172(27.5 \%)$, penicillin antibiotics, and $210(33.5 \%)$ other drug classes (other antibiotics $11.4 \%$, anesthetics $3.5 \%$, corticosteroids $2.9 \%$, opiates $2 \%$, proton pump inhibitors $1.6 \%$, radio contrast agents $0.4 \%$, other drug classes $11.8 \%$. 
gist does not add value to prior suspected by the general doctor. The mean are 4 visits to reach a diagnosis. It is estimated that up to $40 \%$ of the active ingredients could be ruled by provocation test in an initial consultation, only with the evidence of the medical history.

\section{Utility of Component Resolved Diagnosis (CRD) in Illicit Drugs Hypersensitivity}

Illicit drugs can cause allergic sensitization in some drug abusers and atopic patients. We have used component resolved diagnosis (CRD) wit microarrays (ImmunoCAP ${ }^{\circledR}$ and ISAC $^{\circledR}$, Thermo Fisher, Uppsala, Sweden) in patients with response to cannabis sativa and cocaine, diagnosed after positive bronchial challenges [22,23].

The CRD confirmed positivity to lipid-transporter proteins (LTPS), associated to severe symptoms. These reports suggest than cannabis sensitization may be mediated by 2 mechanisms, cross-reactivity (mainly with LTPs and thaumatin-like protein), and exposure-related de novo sensitization. LPTS sensitize primarily through the airways. We characterized the molecular sensitization profile of the diagnoses patients with primary cannabis allergy, who experienced asthma after cannabis or cocaine handling or smoking.

\section{Disclosures}

This study received no external financing. I declare that the authors: Alicia Armentia, Sara Martín and Blanca Martín have participated in the conception, design of the study, analysis and interpretation of the data. Angel San Miguel, Blanca Martin carried out all laboratory analyses and Sara Martin the study of pediatrics patients. All authors have participated in the preparation and critical revision of the paper and all authors have seen and approved the final version of the manuscript. I also declare that no authors have any conflict of interest in connection with this paper.

\section{Acknowledgements}

We thank the nurses and technicians of our hospital for their support.

\section{References}

1. Sociedad española de Alergia e Inmunología Clínica (SEAIC) (2017) Alergológica 2015. Factores epidemiológicos, clínicos y sociosanitarios de las enfermedades alérgicas en España, Faes Farma, Madrid.

2. Hierro B, Mateos J, Cabero T, Moran JA, Armentia A (2016) A predictive model for the diagnosis of allergic drug reactions according to the medical history. J Allergy Clin Immunol Pract 4: 292-300.

3. (2017) Drug allergy, symptoms, diagnosis, treatment \& management. American Academy of Allergy, Asthma \& Immunology.

4. Lieberman P, Nicklas RA, Oppenheimer J, Kemp SF, Lang DM, et al. (2010) The diagnosis and management of anaphylaxis practice parameter: 2010 update. J Allergy Clin Immunol 126: 477-480.

5. Giovanni Satta, Victoria Hill, Marisa Lanzman, Indran Bal- akrishnan (2013) $\beta$-lactam allergy: Clinical implications and costs. Clinical and Molecular Allergy 11: 2-4.

6. Manso L, Sánchez-García S, Ruiz-García M, Arochena L, Andregnette V, et al. (2010) Clinical and Socio-Economical Implications of Drug Allergy Diagnosis in Hospitalized Patients. J Allergy Clin Immunol 125: 158.

7. Caubet JC, Kaiser L, Lemaître B, Fellay B, Gervaix A, et al. (2011) The role of penicillin in benign skin rashes in childhood: A prospective study based on drug rechallenge. $J$ Allergy Clin Immunol 127: 218-222.

8. Classen DC, Pestotnik SL, Evans RS, Lloyd JF, Burke JP (1997) Adverse drug events in hospitalized patients. Excess length of stay, extra costs, and attributable mortality. JAMA 277: 301-306.

9. Solensky R (2012) Allergy to $\beta$-lactam antibiotics. J Allergy Clin Immunol 130: 1442-2.e5.

10. Vallano Ferraz A, Agustí Escasany A, Pedrós Xolvi C, Arnau de Bolós JM (2012) Systematic review of studies assessing the cost of adverse drug reactions. Gac Sanit 26: 277-283.

11. Messaad D, Sahla H, Benahmed S, Godard P, Bousquet J, et al. (2004) Drug provocation tests in patients with a history suggesting an immediate drug hypersensitivity reaction. Ann Intern Med 140: 1001-1006.

12. Demoly P, Kropf R, Bircher A, Pichler WJ (1999) Drug hypersensitivity: Questionnaire. EAACl interest group on drug hypersensitivity. Allergy 54: 999-1003.

13. Doña I, Blanca-López N, Cornejo-García JA, Torres MJ, Laguna JJ, et al. (2011) Characteristics of subjects experiencing hypersensitivity to non-steroidal anti-inflammatory drugs: Patterns of response. Clin Exp Allergy 41: 86-95.

14. Rawlins MD TJ (1991) Mechanisms of adverse drug reactions. ( $4^{\text {th }}$ edn), Oxford.

15. Goben Celi (2003) Drug Allergy. In: Middelton, Middelton's Allergy. ( $7^{\text {th }}$ edn), 1205-1223.

16. Muszkat M (2007) Interethnic differences in drug response: The contribution of genetic variability in beta adrenergic receptor and cytochrome P4502C9. Clin Pharmacol Ther 82: 215-218.

17. Adkinson NF Jr (1984) Risk factors for drug allergy. J Allergy Clin Immunol 74: 567-572.

18. Roujeau JC, Huynh TN, Bracq C, Guillaume JC, Revuz $J$, et al. (1987) Genetic susceptibility to toxic epidermal necrolysis. Arch Dermatol 123: 1171-1173.

19. Hjortlund J, Mortz CG, Skov PS, Bindslev-Jensen C (2013) Diagnosis of penicillin allergy revisited: The value of case history, skin testing, specific IgE and prolonged challenge. Allergy 68: 1057-1064.

20. Doña I, Blanca-López N, Torres MJ, García-Campos J, García-Núñez I, et al. (2012) Drug hypersensitivity reactions: Response patterns, drug involved, and temporal variations in a large series of patients. J Investig Allergol Clin Immunol 22: 363-371.

21. Kowalski ML, Asero R, Bavbek S, Blanca M, Blanca-Lopez $\mathrm{N}$, et al. (2013) Classification and practical approach to the diagnosis and management of hypersensitivity to nonsteroidal anti-inflammatory drugs. Allergy 68: 1219-1232.

22. Armentia A, Herrero M, Martín-Armentia B, Rihs HP, Postigo I, et al. (2014) Molecular diagnosis in cannabis allergy. J Allergy Clin Immun Pract 2: 351-352.

23. Armentia A, Martin-Armentia B, Martín-Armentia S, RuizMuñoz P, Quesada JM, et al. (2018) Cocaine allergy in drug-depenndent patients and allergic people. J Allergy Clin Immunol Pract 6: 201-207. 